\title{
A consistent model for leptogenesis, dark matter and the IceCube signal
}

\author{
M. Re Fiorentin, ${ }^{a}$ V. Niro ${ }^{b, c}$ and N. Fornengo ${ }^{d, e}$ \\ ${ }^{a}$ School of Physics and Astronomy, University of Southampton, \\ SO17 1BJ Southampton, U.K. \\ ${ }^{b}$ Departamento de Física Teórica, Universidad Autónoma de Madrid, \\ Cantoblanco, E-28049 Madrid, Spain \\ ${ }^{c}$ Instituto de Física Teórica UAM/CSIC, \\ Calle Nicolás Cabrera 13-15, Cantoblanco, E-28049 Madrid, Spain \\ ${ }^{d}$ Dipartimento di Fisica, Università di Torino, \\ via P. Giuria, 1, 10125 Torino, Italy \\ eIstituto Nazionale di Fisica Nucleare, Sezione di Torino, \\ via P. Giuria, 1, 10125 Torino, Italy \\ E-mail: m.re-fiorentin@soton.ac.uk, viviana.niro@uam.es, \\ nicolao.fornengo@unito.it
}

ABstract: We discuss a left-right symmetric extension of the Standard Model in which the three additional right-handed neutrinos play a central role in explaining the baryon asymmetry of the Universe, the dark matter abundance and the ultra energetic signal detected by the IceCube experiment. The energy spectrum and neutrino flux measured by IceCube are ascribed to the decays of the lightest right-handed neutrino $N_{1}$, thus fixing its mass and lifetime, while the production of $N_{1}$ in the primordial thermal bath occurs via a freeze-in mechanism driven by the additional $\mathrm{SU}(2)_{R}$ interactions. The constraints imposed by IceCube and the dark matter abundance allow nonetheless the heavier righthanded neutrinos to realize a standard type-I seesaw leptogenesis, with the $B-L$ asymmetry dominantly produced by the next-to-lightest neutrino $N_{2}$. Further consequences and predictions of the model are that: the $N_{1}$ production implies a specific power-law relation between the reheating temperature of the Universe and the vacuum expectation value of the $\mathrm{SU}(2)_{R}$ triplet; leptogenesis imposes a lower bound on the reheating temperature of the Universe at $7 \times 10^{9} \mathrm{GeV}$. Additionally, the model requires a vanishing absolute neutrino mass scale $m_{1} \simeq 0$.

KeYwords: Beyond Standard Model, Cosmology of Theories beyond the SM, Neutrino Physics

ArXiv EPrint: 1606.04445 


\section{Contents}

1 Introduction 1

2 The model 2

$3 \quad N_{1}$ as the dark matter particle $\quad 4$

3.1 Constraints from IceCube 4

3.2 Constraints on the model from $W_{R}$-mediated decays of $N_{1}$

$\begin{array}{lll}3.3 & \text { Relic abundance } & 7\end{array}$

4 The baryon asymmetry 10

$\begin{array}{lll}4.1 \text { Asymmetry production } & 10\end{array}$

$\begin{array}{lll}4.2 & \text { Expressions for the final asymmetry } & 11\end{array}$

5 Conclusions $\quad 14$

\section{Introduction}

The IceCube experiment has so far reported evidence for extraterrestrial high-energy neutrinos [1-4], which cannot be explained by the atmospheric and prompt neutrino components [5-7]. It is possible to relate this signal to astrophysical sources, see for example refs. [8-10] for a general discussion, and references therein, ${ }^{1}$ but it is certainly intriguing to speculate about a new-physics origin of these events. Some models have been proposed, explaining the high-energy IceCube signal as the decay of a long-lived particle $[11,12]$, that can also constitute a viable dark matter (DM) candidate [13-32].

A model of right handed neutrino DM predicting a signal in high energy neutrinos and able to reproduce the matter-antimatter asymmetry with leptogenesis was presented in ref. [23]. Ref. [24] applied a similar framework to interpret the IceCube signal. These are also the aims of this work: as in ref. [23, 24], we will make use of additional heavy right-handed $(\mathrm{RH})$ neutrinos which are responsible for neutrino masses and mixing, via the seesaw mechanism [33-40], and leptogenesis [41]. However, while in ref. [24] the heavy neutrinos are produced by means of inflaton decay, here we will seek for a production mechanism directly from the thermal bath.

We therefore consider a left-right symmetric model (LRSM) [42-45] (see also [46-48] for reviews), with gauge group $\mathrm{SU}(2)_{L} \otimes \mathrm{SU}(2)_{R} \otimes \mathrm{U}(1)_{\tilde{Y}}$ in which three $\mathrm{RH}$ neutrinos $N_{i}$ are naturally accommodated into three $\mathrm{RH}$ doublets of $\mathrm{SU}(2)_{R}$. Among the three heavy neutrinos, we choose the lightest one, $N_{1}$, to be long-lived: the $N_{1}$ lifetime is required to be

\footnotetext{
${ }^{1} \mathrm{~A}$ lot of work has been done in trying to explain the IceCube events in term of astrophysical sources. We refer for example to the references contained in [8-10] for specific discussions.
} 
long enough to make $N_{1}$ a viable dark matter candidate, and at the same time the $N_{1}$ decay width into neutrinos needs to be of the right size to make $N_{1}$ responsible for the IceCube signal. The energy spectrum of the IceCube events (in particular, its end point) and the measured flux therefore determine the mass and the decay width into neutrinos of $N_{1}$. We will show that hadronic decays of $N_{1}$ induce an additional decay channel that can be "fast" enough to be in contradiction with, among others, gamma-ray bounds: this implies that our model needs to assume a "hadrophobic" structure [49, 50], in order to stabilize this decay channel. The IceCube flux implies a severe suppression of the coupling to lefthanded (LH) lepton and Higgs doublets, preventing $N_{1}$ production in the early Universe by means of this type of interactions. Instead, the additional $\mathrm{SU}(2)_{R}$ gauge interactions in the LRSM can provide a viable way to produce $N_{1}$ without spoiling its stability. This is accomplished by means of a freeze-in mechanism [51], tuned to reproduce the present-day DM abundance $\Omega_{N_{1}} h^{2}=\Omega_{\mathrm{DM}} h^{2}=0.1198 \pm 0.0015$ [52]. In fact, we found that the freezeout production mechanism in the context of a LRSM does not lead to the correct relic abundance for PeV-scale DM, since it would require an extremely large entropy dilution. This mechanism has been instead successfully used for keV sterile neutrino DM [53], when sufficient entropy dilution can be achieved $[54,55]$. See instead ref. [56] for a discussion of TeV-scale DM in the context of the LRSM and ref. $[57,58]$ for the freeze-in mechanism in the context of a $\mathrm{keV}$ sterile neutrino DM.

The next-to-lightest $N_{2}$ and the heaviest $N_{3}$ neutrinos in our model are responsible, in turn, for the generation of the matter-antimatter asymmetry of the Universe, via standard thermal leptogenesis. Specifically, we rely on the $N_{2}$-dominated scenario of leptogenesis [59], in which $N_{2}$ 's dynamics is able to produce the correct final baryon asymmetry, measured by the baryon-to-photon ratio $\eta_{B}^{\mathrm{CMB}}=(6.1 \pm 0.1) \times 10^{-10}[52]$. In this case, the thermal production of $N_{2}$ relies on the Yukawa couplings to Higgs and LH doublets, which are not suppressed.

We therefore propose a LRSM in which the lightest heavy neutrino will play the role of the DM particle, while being also responsible, through its decays, for the IceCube signal. At the same time, the other two heavy neutrinos will generate the baryon asymmetry of the Universe via standard thermal leptogenesis.

The paper is organized as follows: in section 2 we present the details of the LRSM model considered in the analysis, in section 3 we focus on the DM particle of the model, the right-handed neutrino $N_{1}$, considering in particular the constraints from IceCube and the relic abundance. In section 4 we present the calculation for the baryon asymmetry and in section 5 we draw our conclusions.

\section{The model}

Considering the standard minimal LRSM [46-48], the RH leptons are fitted into $\mathrm{SU}(2)_{R}$ doublets:

$$
R_{i}=\left(\begin{array}{c}
N_{R i} \\
\ell_{R i}
\end{array}\right) .
$$


To ensure the right spontaneous symmetry breaking pattern and a Majorana mass term for the RH neutrinos, we consider a scalar field $\Delta_{R}$, triplet of $\mathrm{SU}(2)_{R}$, which is responsible for the breaking to the Standard Model (SM) gauge group. The left-right symmetry implies the existence of an $\mathrm{SU}(2)_{L}$ triplet $\Delta_{L}$. The electroweak symmetry breaking (EWSB) is then obtained by exploiting a bi-doublet scalar field $\Phi$. The Yukawa sector then reads:

$$
\mathcal{L}_{Y}=-Y_{i j}^{(1)} \bar{L}_{i} \Phi R_{j}-Y_{i j}^{(2)} \bar{L}_{i} \tilde{\Phi} R_{j}-Y_{i j}^{\Delta}\left(L_{i}^{T} C i \tau_{2} \Delta_{L} L_{j}+R_{i}^{T} C i \tau_{2} \Delta_{R} R_{j}\right)+\text { h.c }
$$

where

$$
\Phi=\left(\begin{array}{cc}
\phi_{1}^{0} & \phi_{1}^{+} \\
\phi_{2}^{-} & \phi_{2}^{0}
\end{array}\right), \quad \Delta_{L, R}=\left(\begin{array}{cc}
\frac{1}{\sqrt{2}} \delta^{+} & \delta^{++} \\
\delta^{0} & -\frac{1}{\sqrt{2}} \delta^{+}
\end{array}\right)_{L, R},
$$

and $\tilde{\Phi} \equiv \tau_{2} \Phi^{*} \tau_{2}$ ( $\tau_{2}$ being the second Pauli matrix).

In order to avoid unwanted low-energy effects due to the $\mathrm{SU}(2)_{R}$ gauge interactions, the $\mathrm{SU}(2)_{R} \otimes \mathrm{SU}(2)_{L} \otimes \mathrm{U}(1)_{\tilde{Y}} \rightarrow \mathrm{SU}(2)_{L} \otimes \mathrm{U}(1)_{Y}$ breaking must take place at very high energies. Therefore, the triplet $\Delta_{R}$ acquires a vacuum expectation value (VEV) $\left\langle\Delta_{R}\right\rangle \equiv v_{R}$ which is suitably large. This implies a Majorana mass matrix for the RH neutrinos given by:

$$
M_{i j}=2 Y_{i j}^{\Delta} v_{R}
$$

which can then be diagonalised to $D_{M}=\operatorname{diag}\left(M_{1}, M_{2}, M_{3}\right)$. We shall assume $M_{\Delta_{R}} \gg M_{i}$. The VEV $v_{R}$ also sets the mass of the $\mathrm{SU}(2)_{R}$ gauge bosons, since $m_{Z_{R}}, m_{W_{R}} \propto v_{R}$.

Labelling the LH lepton doublet with the flavour index $\alpha=e, \mu, \tau$, the Yukawa couplings of the RH neutrinos with the SM Higgs doublet $H$ are given by:

$$
\mathcal{L}_{Y^{\nu}}=-Y_{\alpha i}^{\nu} \bar{L}_{\alpha} H N_{R i}+\text { h.c, }
$$

with

$$
Y^{\nu} \equiv \frac{Y^{(1)} v_{1}+Y^{(2)} v_{2}}{\sqrt{v_{1}^{2}+v_{2}^{2}}},
$$

where $\left\langle\phi_{i}^{0}\right\rangle=v_{i}$. The SM Higgs VEV is obtained as: $\langle H\rangle=\sqrt{v_{1}^{2}+v_{2}^{2}} \equiv v \simeq 174 \mathrm{GeV}$. Finally, we may have that also $\Delta_{L}$ gets a VEV $v_{L} \ll v$. In general, we shall also assume $M_{\Delta_{L}} \gg M_{i}$.

With this symmetry-breaking pattern, the light neutrino masses are generally given by the combination of a type-I and a type-II seesaw terms. We will assume that the type-II seesaw contribution to the light neutrino masses is negligible, or even vanishing if $v_{L}=0$, so that the light neutrino mass matrix is given by:

$$
m_{\nu}=-Y^{\nu} D_{M}^{-1} Y^{\nu T} v^{2}
$$

The light neutrino masses are then obtained through the PMNS mixing matrix $U$ as:

$$
D_{m}=-U^{\dagger} m_{\nu} U^{*}
$$

where $D_{m}=\operatorname{diag}\left(m_{1}, m_{2}, m_{3}\right)$. 
The most relevant interaction of the $\mathrm{RH}$ neutrinos are then given by the Yukawa coupling to Higgs and lepton doublets, in eq. (2.5), and by the $\mathrm{SU}(2)_{R}$ gauge interactions. We will comment in subsection 3.3 on the possible interactions of the RH neutrinos with the $\mathrm{RH}$ triplet $\Delta_{R}$. If we assume the standard inflationary picture of the early Universe, charged leptons and LH neutrinos are part of the thermal bath, hence we can assume that $\mathrm{SU}(2)_{R}$ interactions are able to produce the $\mathrm{RH}$ neutrinos. In the absence of $\mathrm{SU}(2)_{R}$ interactions, the production of the $\mathrm{RH}$ neutrinos is possible only through the Yukawa interactions, which become effective at temperatures around the $\mathrm{RH}$ neutrino mass.

\section{$3 \quad N_{1}$ as the dark matter particle}

\subsection{Constraints from IceCube}

In our model, we assume that the signal detected by IceCube is originated from DM decays. Given the mass pattern, the suitable DM candidate is the lightest heavy neutrino. Therefore, $N_{1}$ will be bound to constitute the whole DM content of the Universe and at the same time produce the IceCube signal.

Let us first analyse the constraints obtained from the IceCube data. $N_{1}$ decays only through the Yukawa couplings of eq. (2.5). The decay channels are:

$$
N_{1} \longrightarrow l_{\alpha}^{\mp} W^{ \pm} ; \quad N_{1} \longrightarrow \nu_{\alpha} Z, \bar{\nu}_{\alpha} Z ; \quad N_{1} \longrightarrow \nu_{\alpha} h, \bar{\nu}_{\alpha} h .
$$

For $M_{1} \gg m_{Z}, m_{h}$, we have monochromatic neutrinos with energy $E_{\nu} \simeq M_{1} / 2$. This will cause a sharp peak and a cutoff in the neutrino energy spectrum, while neutrino cascades will provide a soft tail in the spectrum. From the highest event detected by IceCube [2,60] the DM mass scale can be directly determined [16, 17]: $M_{1} \simeq 4 \mathrm{PeV}$.

It is then possible to estimate the neutrino flux on Earth from the decay of $N_{1}$, assuming it constitutes the total amount of DM in the Universe. By comparing the theoretical prediction to the flux observed by IceCube, once the mass $M_{1}$ is set, the $N_{1}$ 's lifetime $\tau_{N_{1}}$ can be directly derived. From ref. [16, 17, 24], the required lifetime of the DM particles is:

$$
\tau_{N_{1}} \simeq 10^{28} \mathrm{~s} .
$$

Although these are just approximate determinations of the mass and lifetime of $N_{1}$, as inferred from the IceCube data, for the purposes of this paper they can be regarded as a sufficiently good estimation: slight changes in these values will not make any noticeable difference.

The total decay rate $\Gamma_{D 1}=\tau_{N_{1}}^{-1}$ at tree level is given as a function of the Yukawa parameters $Y_{\alpha 1}^{\nu}$ by the expression [61, 62]:

$$
\Gamma_{D 1}=\frac{M_{1}}{8 \pi} \sum_{\alpha}\left|Y_{\alpha 1}^{\nu}\right|^{2} .
$$

Eq. (3.2) implies a constraint on the Yukawa couplings $Y_{\alpha 1}^{\nu}:^{2}$

$$
\sum_{\alpha}\left|Y_{\alpha 1}^{\nu}\right|^{2}=\frac{8 \pi}{M_{1} \tau_{N_{1}}} \ll 1
$$

\footnotetext{
${ }^{2}$ From this result, it is evident that this setup cannot realize strong thermal leptogenesis [63, 64].
} 
Due to the seesaw relation, this constraint will be reflected onto the other Yukawa couplings and the light neutrinos spectrum as well. It is then convenient to introduce the complex orthogonal matrix $\Omega$ parameterisation [65]:

$$
Y^{\nu}=\frac{1}{v} U D_{m}^{1 / 2} \Omega D_{M}^{1 / 2},
$$

such that:

$$
\sum_{\alpha}\left|Y_{\alpha 1}^{\nu}\right|^{2}=\frac{M_{1}}{v^{2}} \sum_{i} m_{i}\left|\Omega_{i 1}\right|^{2}
$$

Hence we obtain:

$$
\sum_{i} m_{i}\left|\Omega_{i 1}\right|^{2}=\frac{8 \pi v^{2}}{M_{1}^{2} \tau_{N_{1}}} \simeq 10^{-52} \mathrm{eV}
$$

Given the light neutrinos mass spectrum with nonzero $m_{2}$ and $m_{3}$, it is clear that, in order to have a vanishing $\sum_{i} m_{i}\left|\Omega_{i 1}\right|^{2}$, we must necessarily have $m_{1} \simeq 0$ and a complex orthogonal matrix of the form:

$$
\Omega \simeq\left(\begin{array}{ccc}
1 & \beta \sin \theta-\alpha \cos \theta & \beta \cos \theta+\alpha \sin \theta \\
\alpha & \cos \theta & -\sin \theta \\
-\beta & \sin \theta & \cos \theta
\end{array}\right)
$$

with $\alpha, \beta, \theta$ complex and $|\alpha|,|\beta| \simeq 0$. All the Yukawa couplings, and hence all the quantities related to the other heavy neutrinos, can then be derived from a complex orthogonal matrix with the form in eq. (3.8) and a fully hierarchical light neutrino spectrum. We also notice that the requirement $m_{1} \simeq 0$ (in particular $m_{1} \ll 10^{-4} \mathrm{eV}$ ) implies that this setup cannot be realised within the so-called $\mathrm{SO}(10)$-inspired leptogenesis models [66-73].

This special form of the complex orthogonal matrix $\Omega$ was pointed out in ref. [23], where a model with one vanishing eigenvalue in the Yukawa matrix was presented. Thereby, the production of the decoupled heavy neutrinos was obtained through active-sterile neutrino oscillations. Alternatively, it can be achieved through inflaton decay [23, 24].

To summarize, by imposing the bound on $N_{1}$ 's lifetime from the IceCube flux has important consequences on the general setup of the model, due to the seesaw relation.

\subsection{Constraints on the model from $W_{R}$-mediated decays of $N_{1}$}

In addition to the decay mode into neutrinos listed in eq. (3.1) that allow an interpretation of the IceCube events, in the simplest realization of the LRSM where both right-handed leptons and right-handed quarks are accommodated in $\mathrm{SU}(2)_{R}$ doublets, $N_{1}$ possesses also an hadronic decay through the mediation of the $W_{R}$ gauge boson into right-handed charged leptons and quarks: $N_{1} \rightarrow l_{R} q_{R} \bar{q}_{R}^{\prime}[74,75]$. The decay rate of this process is: ${ }^{3}$

$$
\Gamma\left(N_{1} \rightarrow l_{R} q_{R} \bar{q}_{R}^{\prime}\right) \simeq \frac{3 g_{R}^{4}}{2^{10} \pi^{3} M_{1}^{3}} \int_{0}^{M_{1}^{2}} d s \frac{M_{1}^{6}-3 M_{1}^{2} s^{2}+2 s^{3}}{\left(s-M_{W_{R}}^{2}\right)^{2}+M_{W_{R}}^{4} \frac{g_{R}^{4}}{(4 \pi)^{2}}} .
$$

\footnotetext{
${ }^{3}$ Note that we found a decay width $\Gamma\left(N_{1} \rightarrow l_{R} q_{R} \bar{q}_{R}^{\prime}\right)$, eq. (3.9), that is a factor 2 smaller than the one reported in ref. [74]. Our total decay width, eq. (3.10), is instead a factor of 3 smaller than the one reported in ref. [75].
} 


\begin{tabular}{|c|c|c|c|c|}
\hline & $\mathrm{SU}(3)_{C}$ & $\mathrm{SU}(2)_{L}$ & $\mathrm{SU}(2)_{R}$ & $\mathrm{U}(1)_{\tilde{Y}}$ \\
\hline$L$ & $\mathbf{1}$ & $\mathbf{2}$ & $\mathbf{1}$ & $-1 / 2$ \\
$Q$ & $\mathbf{3}$ & $\mathbf{2}$ & $\mathbf{1}$ & $1 / 6$ \\
\hline$R$ & $\mathbf{1}$ & $\mathbf{1}$ & $\mathbf{2}$ & $-1 / 2$ \\
$u$ & $\mathbf{3}$ & $\mathbf{1}$ & $\mathbf{1}$ & $2 / 3$ \\
$d$ & $\mathbf{3}$ & $\mathbf{1}$ & $\mathbf{1}$ & $-1 / 3$ \\
\hline
\end{tabular}

Table 1. Multiplet assignment for the hadrophobic LRSM. $L$ and $R$ are fermionic doublets, $Q$ is a quark doublet and $u$ and $d$ stand for the up quark and down quark singlets.

Considering $M_{1} \ll M_{W_{R}}$ and the usual condition $M_{W_{R}}=g_{R} v_{R}$, the total decay width can be cast in this form:

$$
\Gamma\left(N_{1} \rightarrow l_{R} q_{R} \bar{q}_{R}^{\prime}\right)+\Gamma\left(N_{1} \rightarrow \bar{l}_{R} \bar{q}_{R} q_{R}^{\prime}\right) \simeq \frac{3 M_{1}^{5}}{2^{10} \pi^{3} v_{R}^{4}}
$$

where we have also considered $g_{R} \ll 4 \pi$.

This decay rate implies a lifetime for $N_{1}$ larger than the age of the Universe for $v_{R}>5 \times 10^{17} \mathrm{GeV}$. This would reflect into an additional bound on the results we will present in the next sections, nevertheless leaving the standard LRSM viable. However, extrapolations to the $\mathrm{PeV}$ mass range of antiproton bounds on heavy-DM decays [76] and bounds from gamma-rays [22, 77] set much stronger constraints on this decay channel. Even though extrapolations of the knowledge of hadronization processes at such large energies and astrophysical uncertainties are likely present, nevertheless the lifetime associated to this decay channel is plausibly larger than $10^{26} \mathrm{~s}-10^{27} \mathrm{~s}$. This pushes $v_{R}$ in a transplanckian regime, complemented by a suitable requirement $g_{R}<g_{L}$, in order to keep at least the mass of $W_{R}$ below the Planck scale. This solution makes the standard LRSM quite contrived.

Instead, an "hadrophobic" LR choice of the representation for the right-handed quarks prevents the decay of $N_{1}$ through $W_{R}$. This model accommodates the right-handed quarks into singlets of $\mathrm{SU}(2)_{R}$, with a suitable choice of their $\tilde{Y}$ quantum number in order to satisfy the condition $Q=T_{3 L}+T_{3 R}+\tilde{Y}$. The assignments are summarised in table 1 . This model has been considered in the literature in the past, even though for different purposes, see e.g. refs. $[49,50]$. A caveat is that the model should be then embedded in a more complete and final theory to cure the problem of anomalies that are present in the hadrophobic LR model. With the assignments of table 1, the triangle diagram involving three $\mathrm{U}(1)_{Y}$ gauge bosons and the one involving two $\mathrm{SU}(2)_{R}$ and one $\mathrm{U}(1)_{Y}$ gauge bosons are anomalous. A way to cure these anomalies is to add an additional right handed doublet of $\mathrm{SU}(2)_{R}, R^{\prime}$, with $Y_{R}^{\prime}=1 / 2$ and a right handed singlet, $X$, with $Y_{X}=-1$ (plus suitable scalar fields to give mass to these additional fermions). Note that the hadrophobic LR model requires also a doublet to give mass to the quarks in the singlet representation, that cannot couple to the bidoublet. These addition however can be safely applied without affecting the results of our analysis. 


\subsection{Relic abundance}

As mentioned before, we are requiring $N_{1}$ to be the DM particle. We must therefore be able to produce the correct abundance. More specifically, given the current values of $\Omega_{\mathrm{DM}}$, of the critical density $\rho_{c}$ and entropy $s_{0}$, the current DM abundance $Y_{\mathrm{DM}}^{0} \equiv n_{\mathrm{DM}} / s_{0}$ is:

$$
Y_{\mathrm{DM}}^{0}=\frac{\Omega_{\mathrm{DM}} \rho_{c}}{M_{1} s_{0}} \simeq 3.82 \times 10^{-10}\left(\frac{\mathrm{GeV}}{M_{1}}\right) \simeq 9.5 \times 10^{-17},
$$

for $M_{1}=4 \mathrm{PeV}$.

In general, $N_{1}$ can be produced from the thermal bath through its interactions given by the $\mathrm{SU}(2)_{R}$ gauge bosons and the Yukawa couplings in eq. (2.5). We can safely neglect the contribution due to the coupling with $\Delta_{R}$. Indeed, assuming a very high scale $v_{R} \gtrsim 10^{14} \mathrm{GeV}$, while having $M_{1} \simeq 4 \mathrm{PeV}$, implies that the Yukawa couplings $Y^{\Delta}$ for $N_{1}$ are extremely small and the interactions with $\Delta_{R}$ are strongly suppressed. Also the mixing of $N_{1}$ with the other heavy neutrinos gives a negligible contribution [23], as well as the decays $N_{2,3} \longrightarrow N_{1} l_{R} l_{R}^{\prime}$, mediated by $W_{R}$.

The Yukawa interactions with Higgs and lepton doublets give a decay rate at temperature $T$ [79]:

$$
\Gamma_{D 1}(T)=\Gamma_{D 1} \frac{\mathcal{K}_{1}(T)}{\mathcal{K}_{2}(T)},
$$

where $\mathcal{K}_{i}(T)$ are the modified Bessel functions of index $i$.

At the same time, the RH neutrinos are subject to the $\mathrm{SU}(2)_{R}$ gauge interactions, whose scattering rate is given by:

$$
\Gamma_{S}(T) \equiv n_{N_{1}}^{\mathrm{eq}}(T)\langle\sigma|v|\rangle(T),
$$

where $n_{N_{1}}^{\mathrm{eq}}$ is the equilibrium number density of $N_{1}$ and $\langle\sigma|v|\rangle$ is the thermally averaged cross section times velocity. The latter can be estimated via the usual neutrino scattering cross section, as [55]:

$$
\langle\sigma|v|\rangle(T) \simeq G_{F}^{2} T^{2}\left(\frac{m_{W}}{m_{W_{R}}}\right)^{4} \sim G_{F}^{2} T^{2}\left(\frac{m_{W}}{v_{R}}\right)^{4},
$$

where $m_{W}$ is the $W$ boson mass and $G_{F}$ the Fermi constant. Both $\Gamma_{D 1}$ and $\Gamma_{S}$ enter the Boltzmann equations that describe the production of $N_{1}$. To this aim we can consider the variable $z \equiv M_{1} / T$ and the number density $N_{N_{1}}$ of RH neutrinos $N_{1}$, computed in a comoving volume containing one heavy neutrino $N_{1}$ in ultra-relativistic equilibrium. This can be easily related to the abundance $Y_{N_{1}}$. From the definition $N_{N_{1}} \equiv n_{N_{1}}(T) / n_{N_{1}}^{\mathrm{eq}}\left(T \gg M_{i}\right)$, we have $N_{N_{1}}=4 / 3 n_{N_{1}}(T) / n_{\gamma}^{\text {eq }}(T)$, where $n_{\gamma}^{\text {eq }}$ is the equilibrium number density of photons. This is strictly connected to the entropy $s(T)=\pi^{4} g_{*}^{s}(T) n_{\gamma}^{\mathrm{eq}}(T) / 45 \zeta(3)$, such that:

$$
N_{N_{1}}=\frac{4}{135} \frac{\pi^{4} g_{*}^{s}(T)}{\zeta(3)} \frac{n_{N_{1}}(T)}{s(T)} \simeq 2.40 g_{*}^{s}(T) Y_{N_{1}}(T)
$$

In the temperature range of our interest $g_{*}^{s}=g_{*}=112$, considering the three heavy neutrinos as relativistic. 
Using $z$ and $N_{N_{1}}$ we can write [79]:

$$
\frac{d N_{N_{1}}}{d z}=-\left(D_{1}(z)+S(z)\right)\left[N_{N_{1}}(z)-N_{N_{1}}^{\mathrm{eq}}(z)\right]
$$

where $D(z)$ accounts for the decay/inverse-decay processes:

$$
D_{1}(z) \equiv \frac{\Gamma_{D 1}(z)}{H(z) z}
$$

and $S(z)$ for the scattering:

$$
S(z) \equiv \frac{\Gamma_{S}(z)}{H(z) z}
$$

Given eqs. (3.3) and (3.4), $N_{1}$ 's decay rate is strongly suppressed, therefore we can just consider $S(z)$ in the equation for the evolution for $N_{N_{1}}$. Assuming vanishing initial abundance for $N_{1}$ at the end of inflation, i.e. $N_{N_{1}}\left(z_{\mathrm{RH}}\right)=0$ where $z_{\mathrm{RH}}=M_{1} / T_{\mathrm{RH}}$ corresponds to the reheating temperature, we can write the Boltzmann equation for $N_{1}$ as:

$$
\frac{d N_{N_{1}}}{d z}=S(z) N_{N_{1}}^{\mathrm{eq}}(z)
$$

as long as $z<z_{\text {eq }}$, calling $z_{\text {eq }}$ the moment at which $N_{1}$ reaches the equilibrium distribution. Following eq. (3.18), and adopting the expression of the Hubble rate in the radiationdominated epoch [80]:

$$
H(z)=1.66 g_{*}^{1 / 2} \frac{M_{1}^{2}}{M_{\mathrm{Pl}} z^{2}},
$$

with $M_{\mathrm{Pl}}$ being the Planck mass, we can easily find a solution:

$$
N_{N_{1}}\left(z<z_{\mathrm{eq}}\right)=\frac{1}{4} \frac{\zeta(3) G_{F}^{2} M_{\mathrm{Pl}} M_{1}^{3}}{1.66 \pi^{2} \sqrt{g_{*}}}\left(\frac{m_{W}}{v_{R}}\right)^{4}\left(\frac{1}{z_{\mathrm{RH}}^{3}}-\frac{1}{z^{3}}\right),
$$

where we used the expression of $S(z)$ :

$$
S(z)=\frac{3}{2} \frac{\zeta(3) G_{F}^{2} M_{\mathrm{Pl}} M_{1}^{3}}{1.66 \pi^{2} \sqrt{g_{*}}}\left(\frac{m_{W}}{v_{R}}\right)^{4} z^{-4} .
$$

Given the expression of $S(z)$, it is clear that the scattering rate quickly decreases with the temperature, therefore we may expect an intermediate value $\bar{z}$, such that $z_{\mathrm{RH}}<\bar{z}<z_{\text {eq }}$ at which $S(z)$ becomes negligible and the abundance of $N_{1}$ freezes-in, without reaching its equilibrium value. In this way, the current number density of $N_{1}$ is given by:

$$
N_{N_{1}}^{0} \equiv N_{N_{1}}(\bar{z}) \simeq \frac{1}{4} \frac{\zeta(3) G_{F}^{2} M_{\mathrm{Pl}} M_{1}^{3}}{1.66 \pi^{2} \sqrt{g_{*}}}\left(\frac{m_{W}}{v_{R}}\right)^{4} \frac{1}{z_{\mathrm{RH}}^{3}} .
$$

In the left panel of figure 1 we show the ratio $\Gamma_{S}(z) / H(z)$, that measures the efficiency of the scattering reaction. As can be seen from the figure, this ratio is well below one even at the reheating temperature $T_{\mathrm{RH}}$. The abundance of $N_{1}$ as a function of temperature $T$ is shown in the right panel of figure 1. 

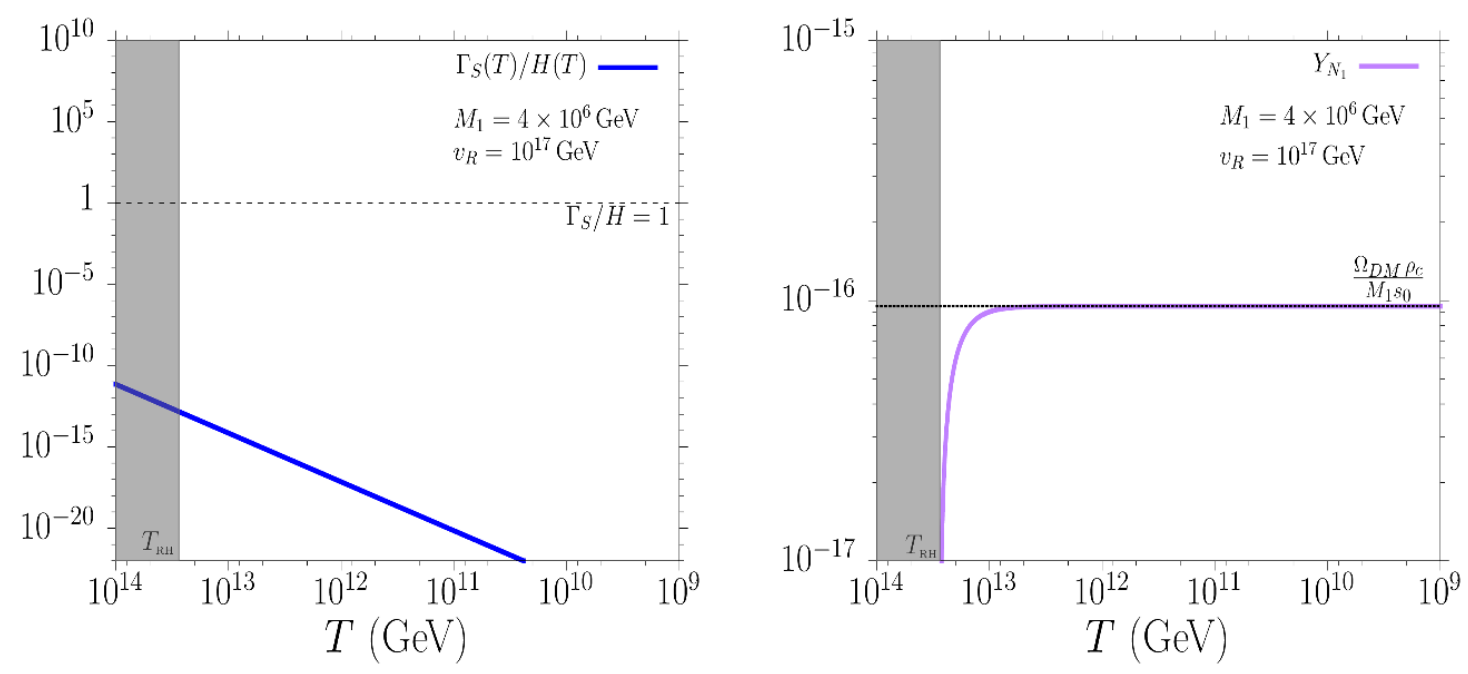

Figure 1. Left panel: evolution in temperature of the ratio $\Gamma_{S} / H$ between scattering rate, eq. (3.13), and the Hubble parameter, eq. (3.20). Right panel: evolution of $N_{1}$ abundance as a function of temperature. We consider $M_{1}=4 \times 10^{6} \mathrm{GeV}, T_{\mathrm{RH}}=2.7 \times 10^{13} \mathrm{GeV}$ and $v_{R}=10^{17} \mathrm{GeV}$.

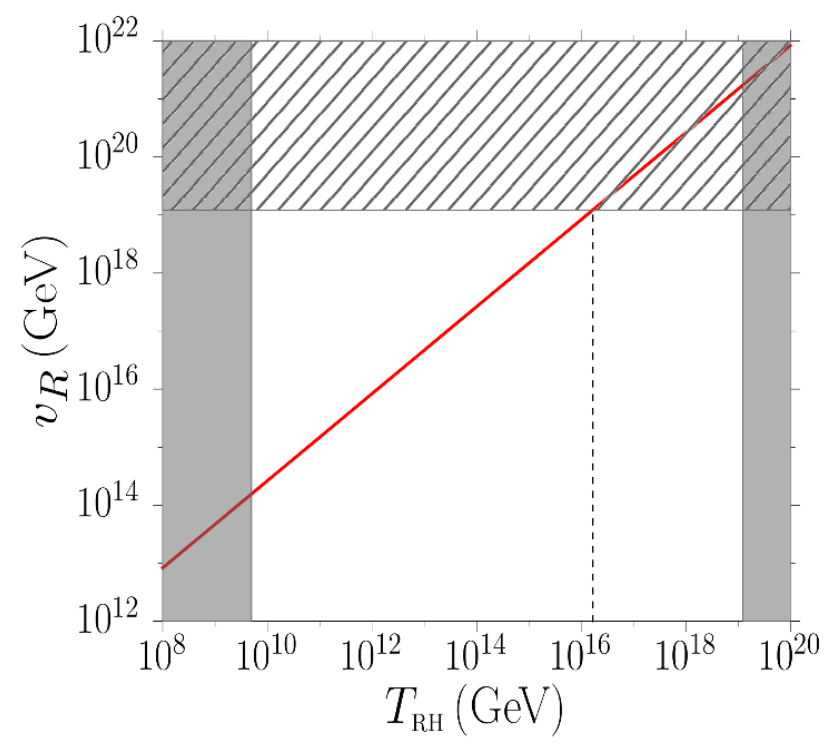

Figure 2. Relation between $v_{R}$ and $T_{\mathrm{RH}}$ that give the correct final abundance $Y_{N_{1}}^{0}=Y_{\mathrm{DM}}^{0}$. The vertical shadowed region on the right corresponds to temperatures above the Planck scale. The upper hatched region is cut out if transplanckian values of $v_{R}$ are excluded. The vertical shaded region on the left shows the lower bound given by leptogenesis, see the discussion in section 4.2 and in particular eq. (4.13).

This model allows for the production of a relatively small abundance of RH neutrinos, in particular of $N_{1}$, exploiting the freeze-in mechanism. The scattering processes mediated by $\mathrm{SU}(2)_{R}$ gauge bosons never become efficient after inflation, since their freeze-out temperature is always higher than $T_{\mathrm{RH}}$. Thus, after reheating, the abundance of $N_{1}$ can only reach a low final value, which is then preserved due to the absence of any other interaction. 
From eq. (3.11), we can obtain a relation between $v_{R}$ and the reheating temperature $T_{\mathrm{RH}}$ such that the final abundance of $N_{1}$ is equal to the current DM abundance. We have:

$$
Y_{N_{1}}^{0} \simeq \frac{1}{4 \times 2.40 g_{*}} \frac{\zeta(3) G_{F}^{2} M_{\mathrm{Pl}}}{1.66 \pi^{2} \sqrt{g_{*}}}\left(\frac{m_{W}}{v_{R}}\right)^{4} T_{\mathrm{RH}}^{3},
$$

and imposing the value in eq. (3.11) we get:

$$
v_{R}=\left[\frac{1}{4 \times 2.40 g_{*}} \frac{\zeta(3) G_{F}^{2} M_{\mathrm{Pl}}}{1.66 \pi^{2} g_{*}^{1 / 2} Y_{\mathrm{DM}}^{0}}\right]^{\frac{1}{4}} m_{W} T_{\mathrm{RH}}^{3 / 4} \simeq 8.3 \times 10^{6} T_{\mathrm{RH}}^{3 / 4},
$$

This relation is a prediction of our model, and it is shown in figure 2 .

\section{The baryon asymmetry}

In our model the other two heavy neutrinos $N_{2}$ and $N_{3}$ are responsible for the production of the correct amount of baryon asymmetry of the Universe. Since we assume that $\Delta_{L}$ does not contribute to the light neutrino masses and that $M_{\Delta_{L}} \gg M_{i}$, our model reduces to ordinary RH neutrino leptogenesis, without any effect from the triplet [62]. For a discussion of the situations in which the scalar triplet could affect leptogenesis we refer to refs. [62, 81].

Given the constraint on $N_{1}$ 's Yukawa couplings in eq. (3.4), that completely decouples the lightest neutrino $N_{1}$, and the fact that $M_{1} \ll 10^{9} \mathrm{GeV}$ [82], the asymmetry must be produced by $N_{2}$. Since $N_{1}$ does not play any role here, we can already expect on $M_{2}$ the same lower bound that applies on $M_{1}$ in $N_{1}$-dominated leptogenesis, i.e. $M_{2} \gtrsim$ $5 \times 10^{8} \mathrm{GeV}[82,83]$. For definiteness, we will always consider hierarchical leptogenesis, obtained by imposing $M_{3} \geq 3 M_{2} \cdot{ }^{4}$ Assuming $M_{2} \gtrsim 5 \times 10^{8} \mathrm{GeV}$, the asymmetry can be produced in two different regimes according to the mass of $N_{2}$ [85-87]: for $5 \times 10^{8} \mathrm{GeV} \lesssim$ $M_{2} \lesssim 5 \times 10^{11} \mathrm{GeV}$ leptogenesis will take place in a two fully-flavoured regime, while for $M_{2} \gtrsim 5 \times 10^{11} \mathrm{GeV}$ leptogenesis will be unflavoured.

\subsection{Asymmetry production}

Case I: $\mathbf{5} \times \mathbf{1 0}^{\mathbf{8}} \mathbf{G e V} \lesssim \boldsymbol{M}_{\mathbf{2}} \lesssim \mathbf{5} \times \mathbf{1 0}^{\mathbf{1 1}} \mathbf{G e V}$ : at temperatures $T \lesssim 5 \times 10^{11} \mathrm{GeV}$ the charged $\tau$-Yukawa interactions are in equilibrium and more efficient than the LH$\mathrm{RH}$ neutrino interactions $[83,88]$. Therefore, the relevant quantities will be the asymmetries $\Delta_{\tau} \equiv B / 3-L_{\tau}$ and $\Delta_{\tau_{2}^{\perp}} \equiv B / 3-L_{\tau_{2}^{\perp}}$, where $\tau_{2}^{\perp}$ defines the flavour component produced by $N_{2}$, orthogonal to the $\tau$ flavour direction. Neglecting ${ }^{5}$ flavour coupling [61, 86, 87, 90-93], scattering terms [79, 83, 94], thermal effects [95] and quantum corrections $[89,96,97]$, the $B-L$ asymmetry produced by $N_{2}, N_{B-L}^{\text {lep, } 2}$, is given by the sum of the produced $\Delta_{\tau}$ and $\Delta_{\tau_{2}^{\perp}}$ asymmetries as [87]

$$
\begin{aligned}
N_{B-L}^{\text {lep }, 2} & =N_{\Delta_{\tau}}^{\text {lep }, 2}+N_{\Delta_{\tau}}^{\text {lep }, 2} \\
& \simeq \varepsilon_{2 \tau} \kappa_{f}\left(K_{2 \tau}\right)+\varepsilon_{2 \tau^{\perp}} \kappa_{f}\left(K_{2 \tau_{2}^{\perp}}\right) .
\end{aligned}
$$

\footnotetext{
${ }^{4}$ Another possibility is provided by resonant leptogenesis (see e.g. ref. [84]), in which at least two heavy neutrinos have a mass splitting comparable to their decay widths. In our situation, this could be applied to $N_{2}$ and $N_{3}$.

${ }^{5}$ The impacts of the corrections we neglect have been estimated not to be larger that about $20 \%[83,89]$.
} 
Here, $\varepsilon_{2 \tau^{\perp}}=\varepsilon_{2 e}+\varepsilon_{2 \mu}$, where $\varepsilon_{2 \alpha}(\alpha=e, \mu, \tau)$ are the $N_{2} C P$ asymmetries in flavour $\alpha$ defined as:

$$
\varepsilon_{2 \alpha} \equiv-\frac{\Gamma_{2 \alpha}-\bar{\Gamma}_{2 \alpha}}{\Gamma_{2}+\bar{\Gamma}_{2}}
$$

where $\Gamma_{2 \alpha}$ and $\bar{\Gamma}_{2 \alpha}$ are the rates of the decays $N_{2} \rightarrow l_{\alpha} H$ and $N_{2} \rightarrow \bar{l}_{\alpha} H$ respectively and $\Gamma_{2}=\sum_{\alpha} \Gamma_{2 \alpha}, \bar{\Gamma}_{2}=\sum_{\alpha} \bar{\Gamma}_{2 \alpha}$. We also introduced the flavoured decay parameters:

$$
\begin{aligned}
K_{i \alpha} & \equiv \frac{\Gamma_{i \alpha}+\bar{\Gamma}_{i \alpha}}{H\left(T=M_{i}\right)} \\
& =\frac{\left|Y_{\alpha i}^{\nu}\right|^{2} v^{2}}{m_{*} M_{i}}=\frac{1}{m_{*}}\left|\sum_{j} \sqrt{m_{j}} U_{\alpha j} \Omega_{j i}\right|,
\end{aligned}
$$

where $m_{*}$ is the equilibrium neutrino mass [79], defined as

$$
m_{*}=\frac{8 \pi v^{2}}{H\left(T=M_{i}\right)}=\frac{16 \pi^{5 / 2} g_{*}^{1 / 2}}{3 \sqrt{5}} \frac{v^{2}}{M_{\mathrm{Pl}}} \simeq 1.08 \times 10^{-3} \mathrm{eV} .
$$

In eq. (4.1) we have $K_{2 \tau_{2}^{\perp}}=K_{2 e}+K_{2 \mu}$, while the $\kappa_{f}\left(K_{2 \alpha}\right)$ are the efficiency factors, whose expressions can be found in the literature [79, 83, 98].

Case II: $M_{2}>\mathbf{5} \times \mathbf{1 0}^{\mathbf{1 1}} \mathrm{GeV}$ : in this case, the flavour interactions are not in equilibrium, therefore the coherence of the lepton and anti-lepton states produced by $N_{2}$ is not broken. Therefore, the evolution of the full $B-L$ asymmetry and $N_{2}$ 's abundance is tracked by the Boltzmann equations, whose solution gives

$$
N_{B-L}^{\mathrm{lep}, 2} \simeq \varepsilon_{2} \kappa_{f}\left(K_{2}\right)
$$

where $K_{2}=\sum_{\alpha} K_{2 \alpha}$ is the total $N_{2}$ 's decay parameter.

We now have an expression for the $B-L$ asymmetry produced by $N_{2}$ 's dynamics, both for Case I and Case II. In order to obtain the final value of the asymmetry we still have to take into account the impact of the processes involving $N_{1}$.

\subsection{Expressions for the final asymmetry}

Below $T \sim M_{2}$, the asymmetry stays constant. However, for temperatures $T \lesssim 5 \times 10^{8} \mathrm{GeV}$ the $\mu$ Yukawa interactions are in equilibrium.

In Case I, this implies that at temperatures $M_{1}<T^{\prime} \lesssim 5 \times 10^{8} \mathrm{GeV}$ the asymmetry $N_{\Delta_{\tau_{2}}}^{\text {lep,2 }}$ gets projected onto the $e$ and $\mu$ flavour directions. Neglecting phantom terms [85, 87], we obtain the asymmetries in $\Delta_{\delta} \equiv B / 3-L_{\delta}(\delta=e, \mu)$, at temperature $T^{\prime}$, simply by

$$
N_{\Delta_{e}}\left(T^{\prime}\right)=\frac{K_{2 e}}{K_{2 \tau_{2}^{\perp}}} N_{\Delta_{\tau_{2}}}^{\mathrm{lep}, 2}, \quad N_{\Delta_{\mu}}\left(T^{\prime}\right)=\frac{K_{2 \mu}}{K_{2 \tau_{2}^{\perp}}} N_{\Delta_{\tau_{2}}}^{\mathrm{lep}, 2} .
$$

The action of $N_{1}$ will then take place along the three flavour directions $e, \mu, \tau$. Considering that the asymmetry produced by $N_{1}$ can be safely neglected, neglecting again flavour 
coupling and taking as initial conditions $N_{\Delta_{e}}, N_{\Delta_{\mu}}$ in eq. (4.6) and $N_{\Delta_{\tau}}$ in eq. (4.1), the Boltzmann equations can be solved, for Case I, giving the total final asymmetry as the sum of the final asymmetries in $\Delta_{\alpha}, \alpha=e, \mu, \tau[71,83,85,87,98]$ :

$$
\begin{aligned}
N_{B-L}^{\mathrm{lep}, \mathrm{f}}= & \sum_{\alpha=e, \mu, \tau} N_{\Delta_{\alpha}}^{\mathrm{lep}, \mathrm{f}} \\
\simeq & \frac{K_{2 e}}{K_{2 \tau_{2}^{\perp}}} \varepsilon_{2 \tau^{\perp}} \kappa_{f}\left(K_{2 \tau_{2}^{\perp}}\right) e^{-\frac{3 \pi}{8} K_{1 e}}+\frac{K_{2 \mu}}{K_{2 \tau_{2}^{\perp}}} \varepsilon_{2 \tau^{\perp}} \kappa_{f}\left(K_{2 \tau_{2}^{\perp}}\right) e^{-\frac{3 \pi}{8} K_{1 \mu}} \\
& +\varepsilon_{2 \tau} \kappa_{f}\left(K_{2 \tau}\right) e^{-\frac{3 \pi}{8} K_{1 \tau}} .
\end{aligned}
$$

As for Case II, the asymmetry in eq. (4.5) gets projected as:

$$
N_{\Delta_{e}}\left(T^{\prime}\right)=\frac{K_{2 e}}{K_{2}} N_{B-L}^{\mathrm{lep}, 2}, \quad N_{\Delta_{\mu}}\left(T^{\prime}\right)=\frac{K_{2 \mu}}{K_{2}} N_{B-L}^{\mathrm{lep}, 2}, \quad N_{\Delta_{\tau}}\left(T^{\prime}\right)=\frac{K_{2 \tau}}{K_{2}} N_{B-L}^{\mathrm{lep}, 2},
$$

and, similarly, the final asymmetry is given by

$$
\begin{aligned}
N_{B-L}^{\mathrm{lep}, \mathrm{f}}= & \sum_{\alpha=e, \mu, \tau} N_{\Delta_{\alpha}}^{\mathrm{lep}, \mathrm{f}} \\
\simeq & \frac{K_{2 e}}{K_{2}} \varepsilon_{2} \kappa_{f}\left(K_{2}\right) e^{-\frac{3 \pi}{8} K_{1 e}}+\frac{K_{2 \mu}}{K_{2}} \varepsilon_{2} \kappa_{f}\left(K_{2}\right) e^{-\frac{3 \pi}{8} K_{1 \mu}} \\
& +\frac{K_{2 \tau}}{K_{2}} \varepsilon_{2} \kappa_{f}\left(K_{2}\right) e^{-\frac{3 \pi}{8} K_{1 \tau}} .
\end{aligned}
$$

As already pointed out, in our specific model, $N_{1}$ 's Yukawa couplings are suppressed. Therefore its washout is negligible and the final asymmetry expressions can be simplified. Indeed, from eqs. (4.3) and (3.4) we have $K_{1 \alpha} \simeq 0$. For this reason, from eq. (4.7), we obtain for Case I:

$$
N_{B-L}^{\text {lep,f }} \simeq \varepsilon_{2 \tau^{\perp}} \kappa_{f}\left(K_{2 \tau_{2}^{\perp}}\right)+\varepsilon_{2 \tau} \kappa_{f}\left(K_{2 \tau}\right),
$$

while for Case II, from eq. (4.9), we get:

$$
N_{B-L}^{\mathrm{lep}, \mathrm{f}} \simeq \varepsilon_{2} \kappa_{f}\left(K_{2}\right) .
$$

We notice that, due to the negligible washout by $N_{1}$, the phantom terms would anyway cancel out, therefore these final expressions are not affected by this correction.

As an example, in figure 3 we consider the case with $M_{2}=10^{11} \mathrm{GeV}$ and a particular choice of the matrix $\Omega$ in eq. (3.8). In the left panel, we show the ratios $\Gamma_{D 2}(z) / H(z)$ and $\Gamma_{I D 2}(z) / H(z)$. These ratios reach a value equal to two at a temperatures between $10^{11}-10^{12} \mathrm{GeV}$. In the right panel, we show the evolution of $N_{2}$ and $B-L$ asymmetry abundances. As can be seen, the $N_{2}$ 's abundance (red line) can reach and track the equilibrium distribution (dashed line), as in the strong-washout regime [79, 87]. The blue line marks the evolution of the asymmetry abundance in modulus. The asymmetry produced at early stages, $T>M_{2}$, is quickly erased at around $T \simeq M_{2}$ when decay processes enter equilibrium. Successively, a new asymmetry is produced with opposite sign and, due to the departure from equilibrium of inverse decay processes, it freezes out to a final value above the experimental bound $Y_{B-L}^{\mathrm{CMB}}$. Therefore, we can conclude that it is possible, in 

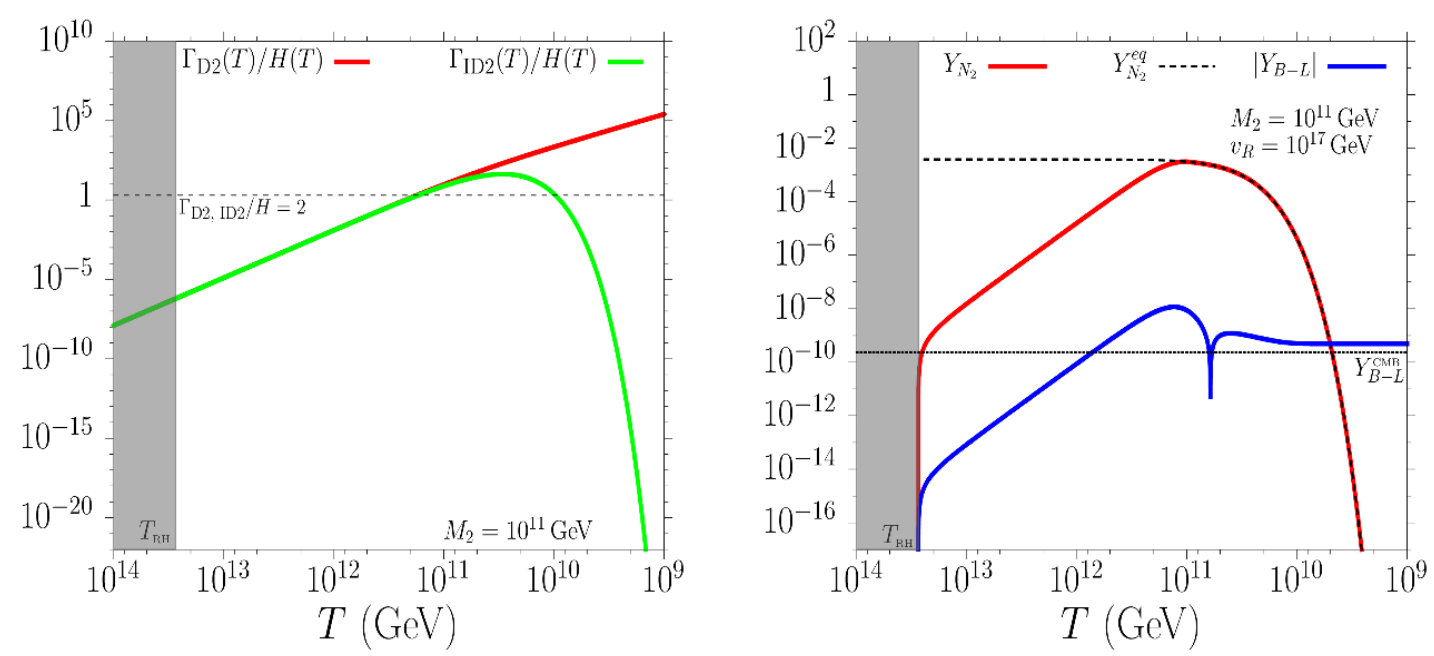

Figure 3. Left panel: evolution in temperature of the ratio between $N_{2}$ 's decay and inverse decay rates and the Hubble parameter. Right panel: evolution of $N_{2}$ and $B-L$ asymmetry abundances as a function of temperature. We consider $M_{2}=10^{11} \mathrm{GeV}, T_{\mathrm{RH}}=2.7 \times 10^{13} \mathrm{GeV}$ and $v_{R}=10^{17} \mathrm{GeV}$. The final $B-L$ asymmetry abundance is obtained as $Y_{B-L}^{\mathrm{CMB}}=\eta_{B}^{\mathrm{CMB}} /\left(2.40 g_{*} 0.96 \times 10^{-2}\right)$, accounting for the sphaleron conversion rate and the dilution factor.

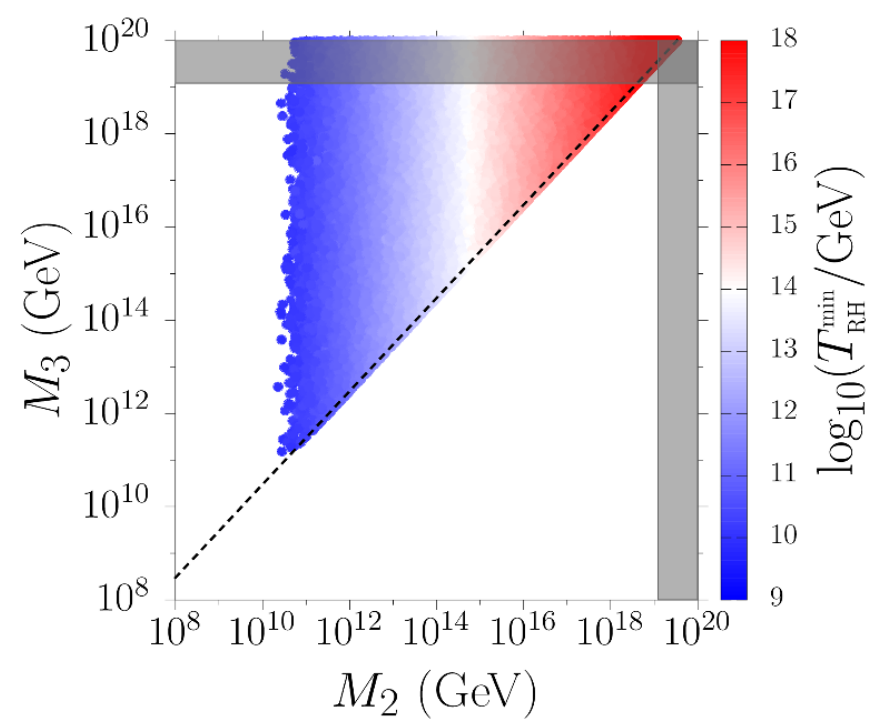

Figure 4. Scatter plot of points in the plane $M_{2}-M_{3}$ that give successful leptogenesis. The colour gives the corresponding $\log _{10}\left(T_{\mathrm{RH}}^{\min } / \mathrm{GeV}\right)$. The dashed line shows the hierarchical limit $M_{3}=3 M_{2}$. The shaded regions exclude masses $M_{i} \geq M_{\mathrm{Pl}}$.

our model, to obtain successful leptogenesis from $N_{2}$ 's decays. Note that in the case shown in the figure, the asymmetry is produced in the two-flavour regime, i.e. Case I eq. (4.10).

In figure 4 a general scan on $M_{2}$ and $M_{3}$, with $M_{3} \geq 3 M_{2}$ is performed and Case I and Case II (eqs. (4.10) and (4.11) respectively) are adopted according to the value of $M_{2}$. Here we assume Normal Ordering (NO) of the light neutrino masses $m_{2}^{2}=m_{1}^{2}+m_{\text {sol }}^{2}$, 
$m_{3}^{2}=m_{1}^{2}+m_{\text {atm }}^{2}$ with $m_{\text {sol }}=0.0087 \mathrm{eV}$ and $m_{\text {atm }}=0.0496 \mathrm{eV}[99] .{ }^{6}$ According to our previous discussion, we set $m_{1}=0$. We scan on the neutrino mixing angles uniformly extracting them in their experimental $3 \sigma$ ranges as in [99], while the Dirac and Majorana phases are extracted on their full variability range. We also scan on the complex angle $\theta$ in $\Omega$, while setting $\alpha=\beta=0$ for simplicity. Moreover, we require $\left|\Omega_{i j}\right|^{2} \leq 2$.

We assume here an instantaneous transition from the two fully-flavoured to the unflavoured regime, at $M_{2}=5 \times 10^{11} \mathrm{GeV}$. A more accurate description should employ a full density matrix formalism [85] to describe leptogenesis in the transition region, however only a small impact is expected. It is also possible to find a lower bound on $M_{2}$ as we expected from considerations similar to $N_{1}$-dominated leptogenesis. In this case the bound appears to be slightly higher: $M_{2} \gtrsim 10^{10} \mathrm{GeV}$.

In figure 4 , the colours encode the minimal reheating temperature $T_{\mathrm{RH}}^{\min }$ needed to produce the correct final asymmetry for each value of $\left(M_{2}, M_{3}\right)$. This is obtained as [79]:

$$
T_{\mathrm{RH}}^{\min } \simeq \frac{M_{2}}{z_{L}\left(\bar{K}_{2}\right)-2 e^{-3 / \bar{K}_{2}}},
$$

where $\bar{K}_{2}=K_{2}$ in Case II, while $\bar{K}_{2}=K_{2 \tau}, K_{2 \tau_{2}^{\perp}}$ in Case I, if the asymmetry in the $\tau$ or $\tau_{2}^{\perp}$ flavour dominates respectively. From figure 4 it is possible to notice that the lowest values are obtained for $M_{2}$ around the lower bound. We obtained a lower bound entirely given by leptogenesis:

$$
T_{\mathrm{RH}}^{\min } \gtrsim 7 \times 10^{9} \mathrm{GeV} .
$$

Using this lower bound on $T_{\mathrm{RH}}^{\min }$ and eq. (3.25), we can find a range of allowed values of $v_{R}$, see also figure 2 . We have $2 \times 10^{14} \mathrm{GeV} \lesssim v_{R} \lesssim 2 \times 10^{21} \mathrm{GeV}$, or the more restrictive case $2 \times 10^{14} \mathrm{GeV} \lesssim v_{R} \lesssim 10^{19} \mathrm{GeV}$ if we require $v_{R}<M_{\mathrm{Pl}}$. We notice that the lower bound on $v_{R}$ agrees with our assumptions of a very high symmetry breaking scale.

\section{Conclusions}

In this paper, we have considered a left-right symmetric model, where a DM particle is produced through a freeze-in process. The model is able to produce the correct final abundance of DM and baryon asymmetry, while at the same time the DM candidate is suitably heavy, $M_{1}=4 \mathrm{PeV}$, and long-lived, $\tau_{N_{1}} \simeq 10^{28} \mathrm{~s}$, to produce high-energy neutrinos consistent with the IceCube signal. It is interesting to point out that this can be realised only with vanishing absolute neutrino mass scale $m_{1} \simeq 0$, which allows for a long lifetime $\tau_{N_{1}}$ through tiny values of $N_{1}$ 's Yukawa couplings. As a consequence, this model predicts a power-law relation between the reheating temperature of the Universe, $T_{\mathrm{RH}}$, and the vacuum expectation value of the $\mathrm{SU}(2)_{R}$ triplet. To obtain successful leptogenesis, a lower bound $T_{\mathrm{RH}} \gtrsim 7 \times 10^{9} \mathrm{GeV}$ should be satisfied.

In conclusion, the model presented in this paper successfully provides a consistent solution to both the DM problem and the generation of the matter-antimatter asymmetry of the Universe through leptogenesis, while producing at the same time a viable interpretation

\footnotetext{
${ }^{6}$ See also ref. [100] for a recent review on neutrino oscillation parameters.
} 
of the highest energy IceCube neutrino events. The basis is a left-right symmetric model, where the right-handed neutrino fields are all involved and mutually necessary in the generation of the different mechanisms at hand (correct DM relic abundance, baryon asymmetry and IceCube neutrino flux). The results can therefore be accommodated naturally in the LRSM scheme.

\section{Acknowledgements}

We thank Bhupal Dev for relevant discussions. MRF acknowledges financial support from the STAG Institute and is also deeply grateful to the Physics Department of the University of Torino for the kind hospitality. VN acknowledges support by Spanish MINECO through project FPA2012-31880, by Spanish MINECO (Centro de excelencia Severo Ochoa Program) under grant SEV-2012-0249. VN acknowledges financial support by the European Union through the ITN ELUSIVES H2020-MSCA-ITN-2015//674896 and the RISE INVISIBLESPLUS H2020-MSCA-RISE-2015//690575. This work is supported by the research grant Theoretical Astroparticle Physics number 2012CPPYP7 under the program PRIN 2012 funded by the Ministero dell'Istruzione, Università e della Ricerca (MIUR) and by the research grant TAsP (Theoretical Astroparticle Physics) funded by the Istituto Nazionale di Fisica Nucleare (INFN).

Open Access. This article is distributed under the terms of the Creative Commons Attribution License (CC-BY 4.0), which permits any use, distribution and reproduction in any medium, provided the original author(s) and source are credited.

\section{References}

[1] ICECube collaboration, M.G. Aartsen et al., First observation of PeV-energy neutrinos with IceCube, Phys. Rev. Lett. 111 (2013) 021103 [arXiv:1304.5356] [INSPIRE].

[2] ICECUBE collaboration, M.G. Aartsen et al., Observation of high-energy astrophysical neutrinos in three years of IceCube data, Phys. Rev. Lett. 113 (2014) 101101 [arXiv: 1405.5303] [INSPIRE].

[3] ICECube collaboration, M.G. Aartsen et al., Evidence for high-energy extraterrestrial neutrinos at the IceCube detector, Science 342 (2013) 1242856 [arXiv:1311.5238] [INSPIRE].

[4] ICECuBE collaboration, M.G. Aartsen et al., Evidence for astrophysical muon neutrinos from the northern sky with IceCube, Phys. Rev. Lett. 115 (2015) 081102 [arXiv:1507.04005] [INSPIRE].

[5] M.V. Garzelli, S. Moch and G. Sigl, Lepton fluxes from atmospheric charm revisited, JHEP 10 (2015) 115 [arXiv:1507.01570] [INSPIRE].

[6] F. Halzen and L. Wille, Upper limit on forward charm contribution to atmospheric neutrino flux, arXiv:1601.03044 [INSPIRE].

[7] F. Halzen and L. Wille, Charm contribution to the atmospheric neutrino flux, Phys. Rev. D 94 (2016) 014014 [arXiv: 1605.01409] [INSPIRE]. 
[8] K. Murase, On the origin of high-energy cosmic neutrinos, AIP Conf. Proc. 1666 (2015) 040006 [arXiv: 1410.3680] [INSPIRE].

[9] L.A. Anchordoqui et al., Cosmic neutrino pevatrons: a brand new pathway to astronomy, astrophysics and particle physics, JHEAp 1-2 (2014) 1 [arXiv:1312.6587] [INSPIRE].

[10] I. Cholis and D. Hooper, On the origin of IceCube's PeV neutrinos, JCAP 06 (2013) 030 [arXiv:1211.1974] [INSPIRE].

[11] Y. Ema, R. Jinno and T. Moroi, Cosmic-ray neutrinos from the decay of long-lived particle and the recent IceCube result, Phys. Lett. B 733 (2014) 120 [arXiv:1312.3501] [INSPIRE].

[12] Y. Ema, R. Jinno and T. Moroi, Cosmological implications of high-energy neutrino emission from the decay of long-lived particle, JHEP 10 (2014) 150 [arXiv:1408.1745] [INSPIRE].

[13] B. Feldstein, A. Kusenko, S. Matsumoto and T.T. Yanagida, Neutrinos at IceCube from heavy decaying dark matter, Phys. Rev. D 88 (2013) 015004 [arXiv:1303.7320] [INSPIRE].

[14] A. Bhattacharya, M.H. Reno and I. Sarcevic, Reconciling neutrino flux from heavy dark matter decay and recent events at IceCube, JHEP 06 (2014) 110 [arXiv:1403.1862] [INSPIRE].

[15] A. Bhattacharya, R. Gandhi and A. Gupta, The direct detection of boosted dark matter at high energies and PeV events at IceCube, JCAP 03 (2015) 027 [arXiv:1407.3280] [INSPIRE].

[16] A. Esmaili and P.D. Serpico, Are IceCube neutrinos unveiling PeV-scale decaying dark matter?, JCAP 11 (2013) 054 [arXiv:1308.1105] [INSPIRE].

[17] A. Esmaili, S.K. Kang and P.D. Serpico, IceCube events and decaying dark matter: hints and constraints, JCAP 12 (2014) 054 [arXiv:1410.5979] [INSPIRE].

[18] Y. Bai, R. Lu and J. Salvado, Geometric compatibility of IceCube TeV-PeV neutrino excess and its galactic dark matter origin, JHEP 01 (2016) 161 [arXiv:1311.5864] [INSPIRE].

[19] K. Murase, R. Laha, S. Ando and M. Ahlers, Testing the dark matter scenario for PeV neutrinos observed in IceCube, Phys. Rev. Lett. 115 (2015) 071301 [arXiv:1503.04663] [INSPIRE].

[20] C.S. Fong, H. Minakata, B. Panes and R. Zukanovich Funchal, Possible interpretations of IceCube high-energy neutrino events, JHEP 02 (2015) 189 [arXiv:1411.5318] [INSPIRE].

[21] J. Kopp, J. Liu and X.-P. Wang, Boosted dark matter in IceCube and at the galactic center, JHEP 04 (2015) 105 [arXiv: 1503.02669] [INSPIRE].

[22] A. Esmaili and P.D. Serpico, Gamma-ray bounds from EAS detectors and heavy decaying dark matter constraints, JCAP 10 (2015) 014 [arXiv: 1505.06486] [INSPIRE].

[23] A. Anisimov and P. Di Bari, Cold dark matter from heavy right-handed neutrino mixing, Phys. Rev. D 80 (2009) 073017 [arXiv: 0812.5085] [INSPIRE].

[24] T. Higaki, R. Kitano and R. Sato, Neutrinoful universe, JHEP 07 (2014) 044 [arXiv: 1405.0013] [INSPIRE].

[25] C. Rott, K. Kohri and S.C. Park, Superheavy dark matter and IceCube neutrino signals: Bounds on decaying dark matter, Phys. Rev. D 92 (2015) 023529 [arXiv:1408.4575] [INSPIRE].

[26] S.M. Boucenna et al., Decaying leptophilic dark matter at IceCube, JCAP 12 (2015) 055 [arXiv: 1507.01000] [INSPIRE]. 
[27] M. Chianese, G. Miele, S. Morisi and E. Vitagliano, Low energy IceCube data and a possible Dark Matter related excess, Phys. Lett. B 757 (2016) 251 [arXiv: 1601. 02934] [INSPIRE].

[28] V. Barger and W.-Y. Keung, Superheavy particle origin of IceCube PeV neutrino events, Phys. Lett. B 727 (2013) 190 [arXiv: 1305.6907] [INSPIRE].

[29] P. Ko and Y. Tang, IceCube events from heavy DM decays through the right-handed neutrino portal, Phys. Lett. B 751 (2015) 81 [arXiv:1508.02500] [INSPIRE].

[30] E. Dudas, Y. Mambrini and K.A. Olive, Monochromatic neutrinos generated by dark matter and the seesaw mechanism, Phys. Rev. D 91 (2015) 075001 [arXiv:1412.3459] [INSPIRE].

[31] P. Di Bari, P.O. Ludl and S. Palomares-Ruiz, Unifying leptogenesis, dark matter and high-energy neutrinos with right-handed neutrino mixing via Higgs portal, arXiv: 1606.06238 [INSPIRE].

[32] P.S.B. Dev, D. Kazanas, R.N. Mohapatra, V.L. Teplitz and Y. Zhang, Heavy right-handed neutrino dark matter and PeV neutrinos at IceCube, JCAP 08 (2016) 034 [arXiv: 1606.04517] [INSPIRE].

[33] P. Minkowski, $\mu \rightarrow$ ey at a rate of one out of $10^{9}$ muon decays?, Phys. Lett. B 67 (1977) 421 [INSPIRE].

[34] T. Yanagida, Horizontal symmetry and masses of neutrinos, Conf. Proc. C7902131 (1979) 95.

[35] M. Gell-Mann, P. Ramond and R. Slansky, Complex spinors and unified theories, Conf. Proc. C 790927 (1979) 315 [arXiv: 1306.4669] [InSPIRE].

[36] S. L. Glashow, The future of elementary particle physics, NATO Sci. Ser. B 61 (1980) 687.

[37] R. Barbieri, D.V. Nanopoulos, G. Morchio and F. Strocchi, Neutrino masses in grand unified theories, Phys. Lett. B 90 (1980) 91 [inSPIRE].

[38] R.N. Mohapatra and G. Senjanović, Neutrino mass and spontaneous parity violation, Phys. Rev. Lett. 44 (1980) 912 [INSPIRE].

[39] J. Schechter and J.W.F. Valle, Neutrino masses in $\mathrm{SU}(2) \otimes \mathrm{U}(1)$ theories, Phys. Rev. D 22 (1980) 2227.

[40] J. Schechter and J.W.F. Valle, Neutrino decay and spontaneous violation of lepton number, Phys. Rev. D 25 (1982) 774.

[41] M. Fukugita and T. Yanagida, Baryogenesis without grand unification, Phys. Lett. B 174 (1986) 45 [INSPIRE].

[42] J.C. Pati and A. Salam, Lepton number as the fourth color, Phys. Rev. D 10 (1974) 275 [Erratum ibid. D 11 (1975) 703] [INSPIRE].

[43] R.N. Mohapatra and J.C. Pati, Left-right gauge symmetry and an isoconjugate model of CP-violation, Phys. Rev. D 11 (1975) 566 [InSPIRE].

[44] R.N. Mohapatra and J.C. Pati, A natural left-right symmetry, Phys. Rev. D 11 (1975) 2558 [INSPIRE].

[45] G. Senjanović and R.N. Mohapatra, Exact left-right symmetry and spontaneous violation of parity, Phys. Rev. D 12 (1975) 1502 [InSPIRE].

[46] W. Grimus, Introduction to left-right symmetric models, in the proceending of the $4^{\text {th }}$ Hellenic School on Elementary particle physics, September 2-20, Corfu, Greece (1992). 
[47] N.G. Deshpande, J.F. Gunion, B. Kayser and F.I. Olness, Left-right symmetric electroweak models with triplet Higgs, Phys. Rev. D 44 (1991) 837 [INSPIRE].

[48] G. Senjanović, Spontaneous breakdown of parity in a class of gauge theories, Nucl. Phys. B 153 (1979) 334.

[49] A. Donini, F. Feruglio, J. Matias and F. Zwirner, Phenomenological aspects of a fermiophobic $\mathrm{SU}(2) \times \mathrm{SU}(2 \times \mathrm{U}(1)$ extension of the standard model, Nucl. Phys. B 507 (1997) 51 [hep-ph/9705450] [INSPIRE].

[50] K. Hsieh, K. Schmitz, J.-H. Yu and C.P. Yuan, Global analysis of general $\mathrm{SU}(2) \times \mathrm{SU}(2) \times \mathrm{U}(1)$ models with precision data, Phys. Rev. D 82 (2010) 035011 [arXiv: 1003.3482] [INSPIRE].

[51] L.J. Hall, K. Jedamzik, J. March-Russell and S.M. West, Freeze-in production of FIMP dark matter, JHEP 03 (2010) 080 [arXiv:0911.1120] [INSPIRE].

[52] Planck collaboration, P.A.R. Ade et al., Planck 2015 results. XIII. Cosmological parameters, Astron. Astrophys. 594 (2016) A13 [arXiv:1502.01589] [INSPIRE].

[53] R. Adhikari et al., A white paper on keV sterile neutrino dark matter, Submitted to: White paper (2016) [arXiv:1602.04816] [INSPIRE].

[54] M. Nemevšek, G. Senjanović and Y. Zhang, Warm dark matter in low scale left-right theory, JCAP 07 (2012) 006 [arXiv: 1205.0844] [INSPIRE].

[55] F. Bezrukov, H. Hettmansperger and M. Lindner, keV sterile neutrino dark matter in gauge extensions of the standard model, Phys. Rev. D 81 (2010) 085032 [arXiv:0912.4415] [INSPIRE].

[56] J. Heeck and S. Patra, Minimal left-right symmetric dark matter, Phys. Rev. Lett. 115 (2015) 121804 [arXiv: 1507.01584] [INSPIRE].

[57] A. Merle, V. Niro and D. Schmidt, New production mechanism for keV sterile neutrino dark matter by decays of frozen-in scalars, JCAP 03 (2014) 028 [arXiv: 1306.3996] [INSPIRE].

[58] A. Merle and M. Totzauer, keV sterile neutrino dark matter from singlet scalar decays: basic concepts and subtle features, JCAP 06 (2015) 011 [arXiv:1502.01011] [INSPIRE].

[59] P. Di Bari, Seesaw geometry and leptogenesis, Nucl. Phys. B 727 (2005) 318 [hep-ph/0502082] [INSPIRE].

[60] ICECube collaboration, M.G. Aartsen et al., The IceCube neutrino observatory Contributions to ICRC 2015 part II: atmospheric and astrophysical diffuse neutrino searches of all flavors, arXiv:1510.05223 [INSPIRE].

[61] S. Davidson, E. Nardi and Y. Nir, Leptogenesis, Phys. Rept. 466 (2008) 105 [arXiv: 0802.2962] [INSPIRE].

[62] T. Hambye and G. Senjanović, Consequences of triplet seesaw for leptogenesis, Phys. Lett. B 582 (2004) 73 [hep-ph/0307237] [INSPIRE].

[63] E. Bertuzzo, P. Di Bari and L. Marzola, The problem of the initial conditions in flavoured leptogenesis and the tauon $N_{2}$-dominated scenario, Nucl. Phys. B 849 (2011) 521 [arXiv: 1007.1641] [INSPIRE].

[64] P. Di Bari, S. King and M. Re Fiorentin, Strong thermal leptogenesis and the absolute neutrino mass scale, JCAP 03 (2014) 050 [arXiv:1401.6185] [INSPIRE]. 
[65] J.A. Casas and A. Ibarra, Oscillating neutrinos and muon $\rightarrow e, \gamma$, Nucl. Phys. B 618 (2001) 171 [hep-ph/0103065] [INSPIRE].

[66] W. Buchmüller and M. Plümacher, Baryon asymmetry and neutrino mixing, Phys. Lett. B 389 (1996) 73 [hep-ph/9608308] [INSPIRE].

[67] F. Buccella, D. Falcone and F. Tramontano, Baryogenesis via leptogenesis in $\mathrm{SO}(10)$ models, Phys. Lett. B 524 (2002) 241 [hep-ph/0108172] [INSPIRE].

[68] E. Nezri and J. Orloff, Neutrino oscillations versus leptogenesis in $\mathrm{SO}(10)$ models, JHEP 04 (2003) 020 [hep-ph/0004227] [INSPIRE].

[69] G.C. Branco, R. Gonzalez Felipe, F.R. Joaquim and M.N. Rebelo, Leptogenesis, CP-violation and neutrino data: What can we learn?, Nucl. Phys. B 640 (2002) 202 [hep-ph/0202030] [INSPIRE].

[70] E.K. Akhmedov, M. Frigerio and A.Yu. Smirnov, Probing the seesaw mechanism with neutrino data and leptogenesis, JHEP 09 (2003) 021 [hep-ph/0305322] [INSPIRE].

[71] P. Di Bari and A. Riotto, Successful type-I Leptogenesis with $\mathrm{SO}(10)$-inspired mass relations, Phys. Lett. B 671 (2009) 462 [arXiv:0809.2285] [INSPIRE].

[72] P. Di Bari and A. Riotto, Testing SO(10)-inspired leptogenesis with low energy neutrino experiments, JCAP 04 (2011) 037 [arXiv:1012.2343] [INSPIRE].

[73] P. Di Bari, L. Marzola and M. Re Fiorentin, Decrypting SO(10)-inspired leptogenesis, Nucl. Phys. B 893 (2015) 122 [arXiv: 1411.5478] [InSPIRE].

[74] P.S. Bhupal Dev, C.-H. Lee and R.N. Mohapatra, Leptogenesis constraints on the mass of right-handed gauge bosons, Phys. Rev. D 90 (2014) 095012 [arXiv:1408.2820] [INSPIRE].

[75] J. Gluza and T. Jeliński, Heavy neutrinos and the $p p \rightarrow l l j j$ CMS data, Phys. Lett. B 748 (2015) 125 [arXiv: 1504.05568] [INSPIRE].

[76] M. Garny, A. Ibarra and D. Tran, Constraints on hadronically decaying dark matter, JCAP 08 (2012) 025 [arXiv: 1205.6783] [INSPIRE].

[77] K. Murase and J.F. Beacom, Constraining very heavy dark matter using diffuse backgrounds of neutrinos and cascaded gamma rays, JCAP 10 (2012) 043 [arXiv:1206.2595] [INSPIRE].

[78] K. Das, T. Li, S. Nandi and S.K. Rai, Diboson excesses in an anomaly free leptophobic left-right model, Phys. Rev. D 93 (2016) 016006 [arXiv:1512.00190] [INSPIRE].

[79] W. Buchmüller, P. Di Bari and M. Plümacher, Leptogenesis for pedestrians, Annals Phys. 315 (2005) 305 [hep-ph/0401240] [INSPIRE].

[80] E.W. Kolb and M.S. Turner, The early universe, Frontiers of Physics, Westview Press (1990).

[81] C. Arina and N. Sahu, Asymmetric inelastic inert doublet dark matter from triplet scalar leptogenesis, Nucl. Phys. B 854 (2012) 666 [arXiv:1108.3967] [INSPIRE].

[82] S. Davidson and A. Ibarra, A lower bound on the right-handed neutrino mass from leptogenesis, Phys. Lett. B 535 (2002) 25 [hep-ph/0202239] [INSPIRE].

[83] S. Blanchet and P. Di Bari, New aspects of leptogenesis bounds, Nucl. Phys. B 807 (2009) 155 [arXiv: 0807.0743] [INSPIRE].

[84] A. Pilaftsis and T.E.J. Underwood, Resonant leptogenesis, Nucl. Phys. B 692 (2004) 303 [hep-ph/0309342] [INSPIRE]. 
[85] S. Blanchet, P. Di Bari, D.A. Jones and L. Marzola, Leptogenesis with heavy neutrino flavours: from density matrix to Boltzmann equations, JCAP 01 (2013) 041 [arXiv: 1112.4528] [INSPIRE].

[86] S. Blanchet and P. Di Bari, Flavor effects on leptogenesis predictions, JCAP 03 (2007) 018 [hep-ph/0607330] [INSPIRE].

[87] S. Antusch, P. Di Bari, D.A. Jones and S.F. King, A fuller flavour treatment of $\mathrm{N}_{2}$-dominated leptogenesis, Nucl. Phys. B 856 (2012) 180 [arXiv:1003.5132] [InSPIRE].

[88] S. Blanchet, P. Di Bari and G.G. Raffelt, Quantum Zeno effect and the impact of flavor in leptogenesis, JCAP 03 (2007) 012 [hep-ph/0611337] [INSPIRE].

[89] C.S. Fong, E. Nardi and A. Riotto, Leptogenesis in the universe, Adv. High Energy Phys. 2012 (2012) 158303 [arXiv:1301.3062] [INSPIRE].

[90] A. Abada, S. Davidson, A. Ibarra, F.X. Josse-Michaux, M. Losada and A. Riotto, Flavour matters in leptogenesis, JHEP 09 (2006) 010 [hep-ph/0605281] [INSPIRE].

[91] E. Nardi, Y. Nir, E. Roulet and J. Racker, The importance of flavor in leptogenesis, JHEP 01 (2006) 164 [hep-ph/0601084] [INSPIRE].

[92] R. Barbieri, P. Creminelli, A. Strumia and N. Tetradis, Baryogenesis through leptogenesis, Nucl. Phys. B 575 (2000) 61 [hep-ph/9911315] [inSPIRE].

[93] W. Buchmüller and M. Plümacher, Spectator processes and baryogenesis, Phys. Lett. B 511 (2001) 74 [hep-ph/0104189] [INSPIRE].

[94] C.S. Fong, M.C. Gonzalez-Garcia and J. Racker, CP violation from scatterings with gauge bosons in leptogenesis, Phys. Lett. B 697 (2011) 463 [arXiv:1010.2209] [INSPIRE].

[95] G.F. Giudice, A. Notari, M. Raidal, A. Riotto and A. Strumia, Towards a complete theory of thermal leptogenesis in the SM and MSSM, Nucl. Phys. B 685 (2004) 89 [hep-ph/0310123] [INSPIRE].

[96] W. Buchmüller and S. Fredenhagen, Quantum mechanics of baryogenesis, Phys. Lett. B 483 (2000) 217 [hep-ph/0004145] [INSPIRE].

[97] A. De Simone and A. Riotto, Quantum Boltzmann equations and leptogenesis, JCAP 08 (2007) 002 [hep-ph/0703175] [INSPIRE].

[98] O. Vives, Flavor dependence of CP asymmetries and thermal leptogenesis with strong right-handed neutrino mass hierarchy, Phys. Rev. D 73 (2006) 073006 [hep-ph/0512160] [INSPIRE].

[99] M.C. Gonzalez-Garcia, M. Maltoni and T. Schwetz, Updated fit to three neutrino mixing: status of leptonic CP-violation, JHEP 11 (2014) 052 [arXiv:1409.5439] [INSPIRE].

[100] M.C. Gonzalez-Garcia, M. Maltoni and T. Schwetz, Global analyses of neutrino oscillation experiments, Nucl. Phys. B 908 (2016) 199 [arXiv:1512.06856] [INSPIRE]. 